\title{
Effect of Carboxyl Graphene on Direct Electrochemistry of Myoglobin and Electrocatalytic Investigation
}

\author{
Wen Zheng ${ }^{1,2}$, Wenshu Zhao ${ }^{1}$, Wei Chen ${ }^{1,2}$, Wenju Weng ${ }^{1,2}$, Zhengwei Liao ${ }^{1}$, Ruixia Dong ${ }^{1}$, \\ Guangjiu Li ${ }^{2}$, Wei Sun ${ }^{1, *}$ \\ ${ }^{1}$ Key Laboratory of Tropical Medicinal Plant Chemistry of Ministry of Education, College of \\ Chemistry and Chemical Engineering, Hainan Normal University, Haikou 571158, P R China \\ ${ }^{2}$ College of Chemistry and Molecular Engineering, Qingdao University of Science and Technology, \\ Qingdao 266042, PR China \\ *E-mail: swyy26@ hotmail.com
}

doi: $10.20964 / 2017.05 .02$

Received: 1 February 2017 / Accepted: 3 March 2017 / Published: 12 April 2017

In this paper the effect of carboxyl graphene (G-COOH) on the electrochemical behavior of myoglobin $(\mathrm{Mb})$ was investigated in detail. A Nafion, $\mathrm{Mb}$ and $\mathrm{G}-\mathrm{COOH}$ modified carbon ionic liquid electrode (CILE) was constructed and applied to electrochemical biosensing. G-COOH with high conductivity and good biocompatibility could act as an electron transfer bridge for enhancing the electron transfer reactivity of $\mathrm{Mb}$. In phosphate buffer solution ( $\mathrm{pH}$ 6.0) $\mathrm{Mb}$ exhibited a pair of good-shape redox peaks on cyclic voltammogram with the formal peak potential $\left(\mathrm{E}^{0^{\prime}}\right)$ as $-0.255 \mathrm{~V}$ (vs. SCE). Electrochemical studies of $\mathrm{Mb}$ were checked with electrochemical parameters calculated. $\mathrm{Mb}$ molecules on the electrode displayed excellent electrocatalytic reduction to trichloroacetic acid (TCA). The current showed a linear response to TCA concentrations from 5.0 to $57.0 \mathrm{mmol} \mathrm{L}^{-1}$ with a low detection limit $\left(1.0 \mathrm{mmol} \mathrm{L}^{-1}\right)$. The Michaelis-Menten constant $\left(K_{M}^{a p p}\right)$ of the fabricated Mb bioelectrode for TCA was determined as $1.30 \mathrm{mmol} \mathrm{L}^{-1}$. Therefore the usage of G-COOH established an effective platform for direct electrochemistry of redox enzymes in the field of third-generation electrochemical sensor.

Keywords: Carbon ionic liquid electrode; Carboxyl graphene; Myoglobin; Direct electrochemistry; Electrocatalysis.

\section{FULL TEXT}

(C) 2017 The Authors. Published by ESG (www.electrochemsci.org). This article is an open access article distributed under the terms and conditions of the Creative Commons Attribution license (http://creativecommons.org/licenses/by/4.0/). 